\title{
Suivi toxicocinétique de la viloxazine (forme à libération prolongée) et du citalopram : étude d'une tentative de suicide
}

\section{Toxicokinetics of viloxazine (slow-release formulation) and citalopram: a case report of non-fatal suicide intoxication}

Benjamin BEAUFILS, Brigitte DELHOTAL-LANDES, Bernard FLOUVAT,

Laboratoire de Toxicologie et de Pharmacocinétique, Hôpital Ambroise Paré 9, avenue Charles de Gaulle - 92104 Boulogne Cedex

*Auteur à qui adresser la correspondance : Benjamin BEAUFILS, Laboratoire de Toxicologie et de Pharmacocinétique, Hôpital Bichat-Claude Bernard, 46, rue Henri Huchard - 75018 Paris Tél : 0614211550 - Fax : 0140258812 - E-mail : benjamin_beaufils@yahoo.fr

(Reçu le 24 juin 2006 ; accepté après modifications le 2 octobre 2006)

\section{RÉSUMÉ}

Une femme de 49 ans est admise dans un service d'urgences médicales pour tentative d'autolyse par ingestion supposée d'une boite de viloxazine (Vivalan (B) forme à libération prolongée (LP) associé à du citalopram (Seropram(B) et diverses benzodiazépines. Durant les heures suivant son admission, elle présente un arrêt cardio-respiratoire. Après restitution de l'activité cardiaque et stabilisation de son état général, elle est transférée dans le service de réanimation médicale de l'hôpital Ambroise Paré. Une analyse toxicologique a alors été effectuée, mettant en évidence la présence de benzodiazépines et l'absence d'antidépresseurs tricycliques et de barbituriques. Une recherche approfondie par. technique Remedi®) (CLHP couplée à un détecteur UV à balayage) révèle la présence de concentrations toxiques de viloxazine à $60 \mathrm{mg} / \mathrm{L}$, ainsi que de citalopram à $1 \mathrm{mg} / \mathrm{L}$ et de desméthyldiazepam à $11 \mathrm{mg} / \mathrm{L}$. Des dosages plasmatiques de viloxazine et de citalopram ont pu être effectués pendant une semaine permettant une analyse toxicocinétique. La demivie d'élimination estimée est, respectivement pour la viloxazine et le citalopram, de 29 et 59 heures. Des cas d'intoxication mortelle ont été observés pour des concentrations inférieures de viloxazine.

MOTS-CLÉS

Viloxazine LP, intoxication aiguë, toxicocinétique.

\section{SUMMARY}

A 49-year-old woman attempted suicide with about $4 \mathrm{~g}$ viloxazine slow-release form (Vivalan(B), citalopram (Seropram(B) and various benzodiazepines. Conscious, she was admitted in urgency department and few hours later she had a respiratory-heart attack. After restoration of the cardiac activity and stabilization, she is transferred in an intensive care unit to the Hospital Ambroise Paré. The patient was in coma and respiratory insufficiency. With its admission, the toxicological assessment points out the presence of benzodiazepines, the absences of tricyclic antidepressants and barbiturates. A toxicological screening by Remedi(B) (an. automated HPLC system associated with a full scan $U V$ detector) highlights plasma concentrations of viloxazine at $60 \mathrm{mg} / \mathrm{l}$, citalopram at $1 \mathrm{mg} / \mathrm{l}$ and desmethyldiazepam at $11 \mathrm{mg} /$, considered as toxic concentration for the 3 drugs. Plasma determination of viloxazine and citalopram could be carry on during one week allowing a toxicokinetic analysis. A 2 to 4 fold increase of the elimination half-life is observed for citalopram and viloxazine, with $59 \mathrm{~h}$ and $29 \mathrm{~h}$ respectively. Some fatal intoxication is already reported for less viloxazine-plasma-concentration and no one for the slow release form.

\section{KEY-WORDS}

Slow release form viloxazine, self-poisoning, toxicokinetics. 


\section{Introduction}

La recherche des antidépresseurs tricycliques est demandée de manière systématique par les services des urgences médicales et de réanimation lors de bilans toxicologiques initiaux. Les méthodes immunologiques rapides, FPIA (Fluorescence Polarization ImmunoAssay) et EMIT (Enzyme Multiplied Immuno Technique), ne permettent que la recherche des antidépresseurs de la famille des tricycliques tels que les imipraminiques ou les triptylines. Les antidépresseurs non tricycliques échappent à cette recherche toxicologique de première intention. Seules des méthodes chromatographiques plus complexes permettent la mise en évidence de l'ensemble des antidépresseurs mais celles-ci ne sont jamais réalisées de manière systématique en urgence. La toxicité cardiaque des antidépresseurs tricycliques a déjà été largement démontrée (1-4). La classe des nouveaux antidépresseurs est, quant à elle de plus en plus utilisée pour sa moindre toxicité cardiaque (5). Dans notre cas, la poly-intoxication médicamenteuse associe plusieurs médicaments : la viloxazine forme à libération prolongée (LP), le citalopram et des benzodiazépines, qui ont été prescrits à la patiente pour traiter son syndrome dépressif, son anxiété et ses troubles du sommeil. La viloxazine est un antidépresseur bicyclique non-imipraminique et non-IMAO ne possédant aucune propriété sédative (Figure 1a). Il présente une faible toxicité à dose thérapeutique et peu d'effet épileptogène (6-9). Ce médicament est actif dans le traitement des dépressions majeures et possède également des propriétés psychoanaleptiques. Il présente une activité bloquante de la recapture de la noradrénaline, de la dopamine et de la sérotonine $(10,11)$. Cependant, le mécanisme de l'activité anti-dépressive n'est pas complètement élucidé. Son action anti-dépressive est presque totalement dépourvue de composantes anticholinergiques et antihistaminiques. Il est commercialisé en France en comprimés à $100 \mathrm{mg}$ et $300 \mathrm{mg}$ LP (Vivalan®), AztraZeneca, Rueil-Malmaison, France). La posologie recommandée est de 100 à $300 \mathrm{mg} / \mathrm{j}$ avec une augmentation progressive en fonction de la tolérance clinique (posologie maximale de $600 \mathrm{mg} / \mathrm{j}$ ). Le citalopram (comprimés
10 et $20 \mathrm{mg}$, Seropram ${ }^{\circledR}$, Lundbeck, Paris, France) est un antidépresseur sérotoninergique de la classe des isobenzofuranes (figure1b) $(6,7,12)$. Inhibiteur spécifique de la recapture de la sérotonine, il ne possède que peu ou pas d'affinité pour les autres récepteurs habituels notamment cholinergiques, histaminergiques ou adrénergiques. Cette sélectivité pourrait expliquer la faible incidence de certains effets indésirables du citalopram comme les syndromes sérotoninergiques (troubles neurologiques, neurovégétatifs et respiratoires), des sédations et des syndromes extrapyramidaux (dyskinésies, hyperthermie, hypertonie, hyper-réflexie, tachycardie) (13). Il est indiqué dans les dépressions modérées à sévères, la phobie sociale, les attaques de panique ne présentent pas de phénomène de tolérance. La posologie recommandée est de 20 à $60 \mathrm{mg} / \mathrm{j}$ en fonction de la réponse clinique. A dose unique, chez le sujet sain, les paramètres pharmacocinétiques (14-17) de ces deux molécules présentent des différences importantes liées à leurs caractéristiques galéniques (Tableau I). La viloxazine et le citalopram sont rapidement et complètement absorbés au niveau intestinal avec une biodisponibilité de 0,6 à 0,95 pour la viloxazine et de 0,72 pour le citalopram. Ces deux molécules subissent un métabolisme hépatique important (hydroxylation et glucuroconjugaison) mais la forme inchangée reste prédominante. La concentration plasmatique maximale est obtenue entre 3 et $9 \mathrm{~h}$ pour la viloxazine et 2 à $4 \mathrm{~h}$ pour le citalopram. La demi-vie d'élimination est de $5,5 \mathrm{~h}$ et d'environ $33 \mathrm{~h}$ respectivement pour la viloxazine et le citalopram. L'élimination rénale est prédominante pour la viloxazine à l'inverse du citalopram éliminé majoritairement par voie hépatique $(85 \%)$. Bien que connue depuis plus d'une dizaine d'années, de rares cas d'intoxications par la viloxazine seule ou en association avec d'autres antidépresseurs ont été décrits. Cependant, seules les concentrations plasmatiques de viloxazine à l'admission ont été quantifiées et peu de données toxicocinétiques ont été décrites lors d'intoxications aiguës avec sa forme LP. Nous rapportons ici l'observation d'un cas d'intoxication volontaire aiguë non mortelle, pour laquelle la toxicocinétique de la viloxazine LP et du citalopram a été évaluée.

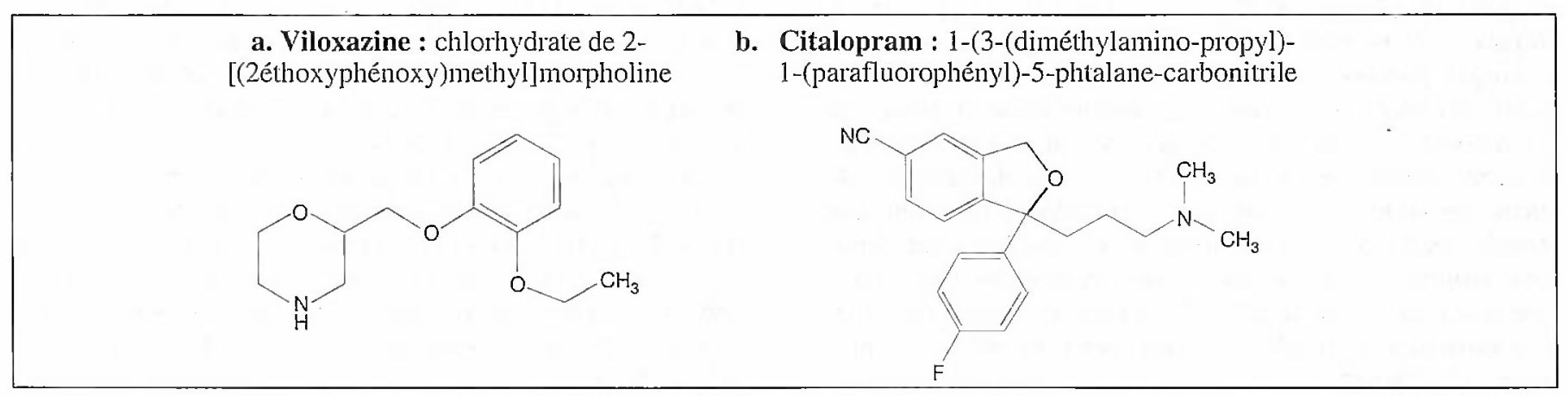

Figure 1 : a. Structure chimique de la viloxazine, Vivalan ${ }^{\oplus}$ : chlorhydrate de 2-[(2éthoxyphénoxy)methyl]morphine. b. Structure chimique du citalopram, Seropram ${ }^{\oplus}$ : 1-(3-(diméthylanino-propyl)-1-(parafuorophényl)-5-phtalane-carbonitrile. 
Tableau I : Paramètres pharnacocinétiques de la viloxazine forme à libération prolongée et du citalopram.

\begin{tabular}{|l|c|c|c|c|c|c|c|}
\hline & $\begin{array}{c}\text { Tmax } \\
(\mathbf{h})\end{array}$ & $\begin{array}{c}\text { Cmax } \\
(\mathbf{m g} / \mathbf{l})\end{array}$ & $\mathbf{F}^{*}$ & $\begin{array}{c}\mathbf{V d} / \mathbf{F} \\
(\mathbf{1} / \mathbf{k g})\end{array}$ & $\begin{array}{c}\text { Métabolisme } \\
\text { hépatique }\end{array}$ & $\begin{array}{c}\text { Demi-vie } \\
(\mathbf{h})\end{array}$ & Référence \\
\hline Viloxazine LP & $3-9$ & 2,3 & $0,6-0,95$ & 1,6 & +++ & $6-7$ & 14,17 \\
Citalopram & $2-4$ & & 0,8 & 14 & +++ & 33 & 15,16 \\
\hline
\end{tabular}

* F = biodisponibilité

\section{Matériels et méthodes Description du cas clinique}

Une femme de 49 ans est admise aux urgerices médicales d'un hôpital des Hauts-de-Seine pour une récidive de tentative d'autolyse. Au cours des mois précédant sa tentative, elle y était suivie pour alcoolisme chronique et syndrome dépressif. Au vu des conditionnements retrouvés par les pompiers, elle aurait ingéré de la viloxazine LP associé à du citalopram et des benzodiazépines. La patiente arrive agitée et consciente (score de Glascow à 13) et ne présente pas de syndrome de choc. Elle a une tension artérielle normale $(120 / 90 \mathrm{~mm} / \mathrm{Hg}$ ), aucun trouble cardiaque (ECG normal) et un état hémodynamique satisfaisant. L'auscultation cardio-respiratoire ne révèle pas d'anomalie et les réflexes ostéo-tendineux sont positifs. Aucune recherche de toxiques ni aucun traitement épurateur ou évacuateur n'est alors initié. Elle reste en observation dans le service porte des urgences. Dans les heures suivant son admission, la patiente présente un arrêt cardio-respiratoire avec perte de conscience, arrêt de la respiration et un pouls carotidien à peine perceptible. Une réanimation cardio-pulmonaire est entreprise : l'activité cardiaque est restituée après un massage externe, l'administration d'adrénaline par voie intraveineuse (un bolus de $1 \mathrm{mg}$ répété puis une perfusion continue à $0,5 \mathrm{mg} / \mathrm{h}$ ), une intubation et une ventilation artificielle. Seul un bilan biologique classique est demandé (enzymes cardiaques, ionogrammes, gaz du sang, glycémie). Malgré la réanimation pratiquée, la patiente reste dans un coma profond, n'a plus de réaction à la douleur et présente une tachycardie transitoire (tension diastolique à $19 \mathrm{mmHg}$ ). Elle est alors transférée, par le SAMU dans le service de réanimation médicale de l'hôpital Ambroise Paré (Boulogne-Billancourt, France) toujours dans le coma et en insuffisance respiratoire. Les analyses toxicologiques sanguines effectuées à son admission en réanimation, soit plus de $24 \mathrm{~h}$ après l'ingestion des médicaments, sont positives pour les benzodiazépines et négatives pour les barbituriques et les antidépresseurs tricycliques par techniques immunologiques (EMITß, Dade Behring, France). L'alcoolémie, recherchée par technique de chromatographie en phase gazeuse couplée à un détecteur à ioni- sation de flamme, s'avère inférieùre à la limite de quantification $(0,1 \mathrm{mg} / \mathrm{l})$. Une recherche toxicologique approfondie est alors réalisée à l'aide de la technique Remedi ${ }^{\circledR}$ mettant en évidence la présence de viloxazine, de citalopram et de desméthyldiazépam. Une réanimation classique est débutée avec administration d'adrénaline pendant 3 jours et une surveillance de la fonction cardio-respiratoire ne présentant pas de troubles apparents. Pour pallier son insuffisance respiratoire aiguë, la patiente reste intubée et ventilée. Six jours plus tard, un anti-épileptique lui est administré pour lutter contre l'apparition de myoclonies (Phénobarbital, Gardénal@, posologie de $200 \mathrm{mg} / \mathrm{j}$ avec des concentrations plasmatiques comprises entre $16,2$ et $27,9 \mathrm{mg} / \mathrm{l})$. L'évolution favorable de la patiente permet une extubation et le retour à un état de conscience après un mois passé dans le service de réanimation médicale. Elle est transférée dans l'hôpital d'origine en service de soins intensifs où elle présente une encéphalopathie post-anoxique sévère avec des myoclonies généralisées. Compte tenu du contexte clinique, la viloxazine et le citalopram ont été dosés, sur 13 prélèvements sanguins successifs, de la $1^{\text {ere }}$ à la $168^{\text {tint }}$ heure d'hospitalisation en réanimation médicale de l'hôpital Ambroise Paré.

\section{Méthode de dosage}

Les concentrations plasmatiques des trois molécules ont été déterminées par chromatographie liquide haute performance associée à un détecteur UV à balayage (Remedi $\AA$, Biorad, France). Le pré-traitement des échantillons ( $1 \mathrm{ml}$ de sérum) consiste en une dilution avec $200 \mu \mathrm{l}$ de la solution d'étalons internes et une simple filtration par centrifugation. Comme les molécules amphotères ou peu basiques sont éluées des colonnes de séparation rapidement, et plus tardivement pour les composés basiques, deux étalons internes sont utilisés pour vérifier le bon comportement chromatographiques des colonnes : le $\mathrm{N}$-éthyl-diazépam et la chlorpheniramine. Après centrifugation, les échantillons sont déposés sur le carrousel d'injection. La programmation de l'analyse est immédiate. La préparation de l'échantillon est suivie par une extraction automatisée en série sur colonne de purification hydrophobe qui 
retient les médicaments de différentes polarités et élimine les protéines, les sels minéraux et les acides carboxyliques hydrophiles. Après élution, le solvant de la phase mobile est alors introduit et entraine l'éluat dans une colonne échangeuse d'anions (colonne d'extraction) qui retient les acides organiques endogènes. Le flux est alors dirigé en mode isocratique vers deux colonnes séparatives de polarités différentes. Une colonne $\mathrm{C} 18$ polaire (colonne de séparation I) et une colonne échangeuse de cations faibles (colonne de séparation II) permettent de différencier les composés basiques. L'identification des molécules est effectuée par un détecteur UV à balayage couplé à un logiciel d'algorithme. Le balayage des longueurs d'ondes s'effectue entre 205 et $235 \mathrm{~nm}$. Les spectres des échantillons sont alors automatiquement comparés à ceux sauvegardés dans la librairie du logiciel. Le temps de rétention, le spectre d'absorption moléculaire dans I'UV et la comparaison à une banque de données permettent, à l'aide d'algorithmes mathématiques d'identifier les principaux toxiques dont la viloxazine et le citalopram. Un calcul de concentration est également possible, et le facteur, reliant la hauteur du pic à la concentration de la substance, a été calculé par Biorad à partir des analyses de solutions pures. Ce facteur, pouvant varier légèrement en fonction des colonnes analytiques utilisées, ne permet qu' un dosage dit semiquantitatif. Le bon fonctionnement de l'appareil est testé chaque jour avec une solution témoin (diazépam, morphine, imipramine, amphétanine et hydrocodone). L'analyse chromatographique dure 16 min et l'expertise du tracé 3 à 4 min. Cette technique permet l'identification de 900 molécules en une seule analyse.

\section{Traitement des données et analyse phar- macocinétique}

L'analyse pharmacocinétique des concentrations plasmatiques en fonction du temps de viloxazine LP et du citalopram est réalisée à l'aide du logiciel siphar(ß (Pharsight, France). Les paramètres pharmacocinétiques déterminés sont : (1) la concentration plasmatique maximale (Cmax) ; (2) le temps nécessaire pour atteindre la concentration plasmatique maximale (Tmax); (3) l'aire sous courbe du temps 0 à t, temps de la dernière concentration quantifiable après administration (AUC $0 \rightarrow \mathrm{t}$ ) ; (4) la clairance totale apparente $\mathrm{Cl} / \mathrm{F}$ $=$ dose $/ \mathrm{AUC}$ ( $\mathrm{F}=$ biodisponibilité absolue); (5) le volume de distribution apparent $\mathrm{Vd} / \mathrm{F}=\operatorname{dose} /(\mathrm{kexAUC})(\mathrm{ke}$ = constante d'élimination) ; (6) la demi-vie apparente $\mathrm{d}^{\prime}$ élimination $=0,693 / \mathrm{ke}$. L'aire sous courbe est calculée en utilisant la méthode log- et linéaire des trapèzes dans la phase ascendante et descendante respectivement. Les données de la viloxazine LP ont été analy- sées à l'aide d'un modèle à un compartiment utilisant une équation bi-exponentielle du type : $\mathrm{Cp}=\mathrm{B} \cdot \mathrm{e}^{*}(-$ $\left.\mathrm{ke}{ }^{*} \mathrm{t}\right)-\mathrm{A} \cdot \mathrm{e}^{*}\left(-\mathrm{ka} \mathrm{a}^{*} \mathrm{t}\right)$ où $\mathrm{Cp}$ est la concentration plasmatique de viloxazine au temps $t$, ke et ka sont les constantes du premier ordre d'élimination et d'absorption. Il en est de même pour la cinétique du citalopram pour laquelle nous avons utilisé un modèle à deux compartiments caractérisé par une équation à trois exponentielles du type : Cp = B.e.* $\left(-\mathrm{ke}^{*} \mathrm{t}\right)+\mathrm{C} . \mathrm{e}^{* *}(-\mathrm{ke} 2 * \mathrm{t})$ -A.e* $\left(-\mathrm{ka}^{*} \mathrm{t}\right)$

\section{Résultats}

Les concentrations plasmatiques en fonction du temps des trois molécules incriminées dans l'intoxication de la patiente depuis son admission en réanimation à l'hôpital Ambroise Paré jusqu'au temps $\mathrm{t}=168 \mathrm{~h}$ sont regroupées sur la Figure 2. Le délai entre l'ingestion des médicaments et le premier prélèvement étant très important (environ trente h) seules, les cinétiques d'éliminations de la viloxazine et du citalopram peuvent être déterminées.

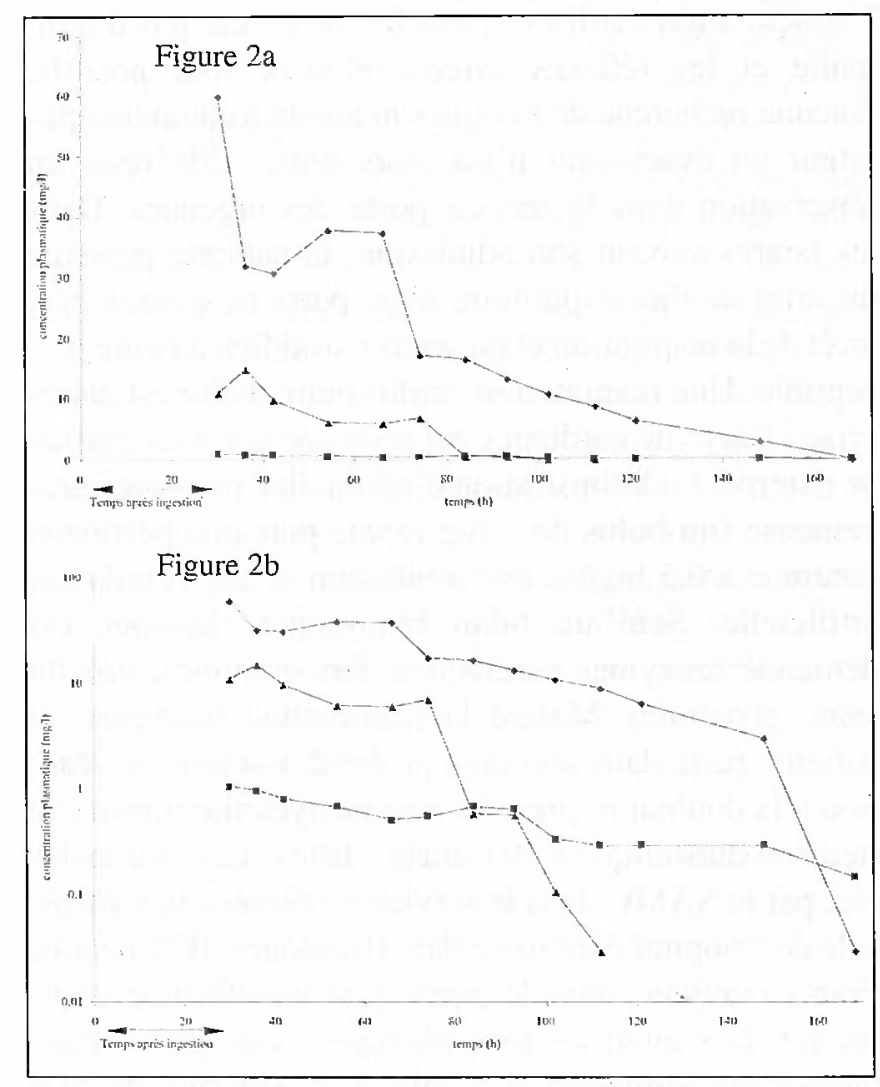

Figure 2 : a. Évolution des concentrations plasmatiques en fonction du temps de la viloxazine LP $(\diamond)$, du citalopram ( et du desméthyldiazépan( $\Delta$ ) entre le temps $t=30 \mathrm{~h}$ et le temps $t=168 \mathrm{~h}$.

b. Evolution des concentrations plasmatiques en fonction du

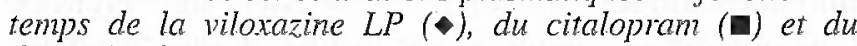
desméthyldiazépam( $\mathbf{\Delta}$ ) entre le temps $t=30 \mathrm{~h}$ et le temps $t=168$ h en coordonnée semi-logarithmique. 
Les premières concentrations plasmatiques quantifiées sont de $60 \mathrm{mg} / \mathrm{l}$ pour la viloxazine, de $1 \mathrm{mg} / \mathrm{l}$ pour le citalopram et de $11 \mathrm{mg} / \mathrm{l}$ pour le desméthyldiazépam. Nous mettons donc en évidence des concentrations plasmatiques élevées puisque 5 à 10 fois supérieures aux concentrations thérapeutiques. Elles sont de $0,5 \mathrm{mg} / \mathrm{l}$ à $5 \mathrm{mg} / \mathrm{l}$ pour la viloxazine et de 0,03 à 0,3 $\mathrm{mg} / \mathrm{l}$ pour le citalopram et la concentration toxique de viloxazine est de $45 \mathrm{mg} / \mathrm{l}(18,19)$. Il n'y a pas de réelle concentration toxique définie pour le citalopram. Les paramètres pharmacocinétiques ont été évalués en prenant en compte un délai de trente heures entre l'ingestion et le premier prélèvement effectué dans le service de réanimation médicale (tableau 2 ). Le modèle pharmacocinétique de la viloxazine et du citalopram (figure 3) permet de prédire la phase d'absorption de la molécule et les paramètres pharmacocinétiques la caractérisant ( $\mathrm{Cmax}$ et Tmax). La présence d'une concentration nulle au temps $t=0$, sur les figures $3 a$ et $3 \mathrm{~b}$, est nécessaire pour que le logiciel puisse converger correctement vers le modèle pharmacocinétique. Nous pouvons observer une bonne prédiction du modèle pour la phase d'élimination de chacune des 2 molécules.

\section{Discussion}

Parmi les cas d'intoxications aiguës par les antidépresseurs tel que la viloxazine ou le citalopram décrits dans la littérature (20-22), aucun n'implique la forme LP de la viloxazine. Les signes cliniques les plus classiques liés à un surdosage en viloxazine sont un coma, une agitation, des myoclonies généralisées, une hypotension et une tachycardie sinusale. Le citalopram, pour des doses supérieures à $1,5 \mathrm{~g}$ entraîne des convulsions, un allongement de l'espace QT et QRS, des troubles du rythme ventriculaire et un syndrome sérotoninergique. Les signes cliniques que nous avons rencontrés sont comparables à ceux décrits dans la littérature (20-24)
Figure 3a

\section{Viloxazine}

Concentration (mg/)

100

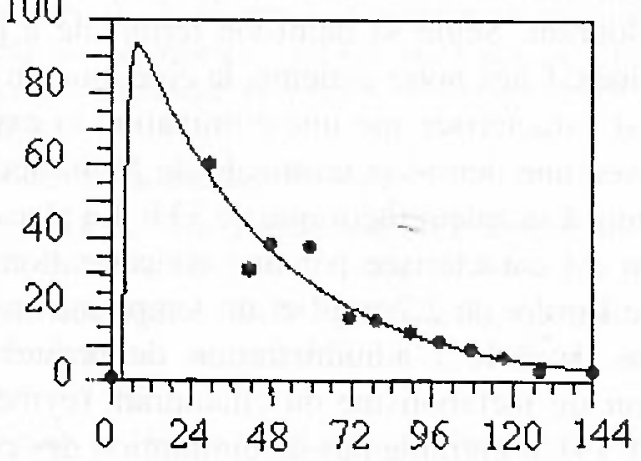

\section{Temps $(h)$}

Figure $3 b$

\section{Citalopram}

Concentration (mg/1)

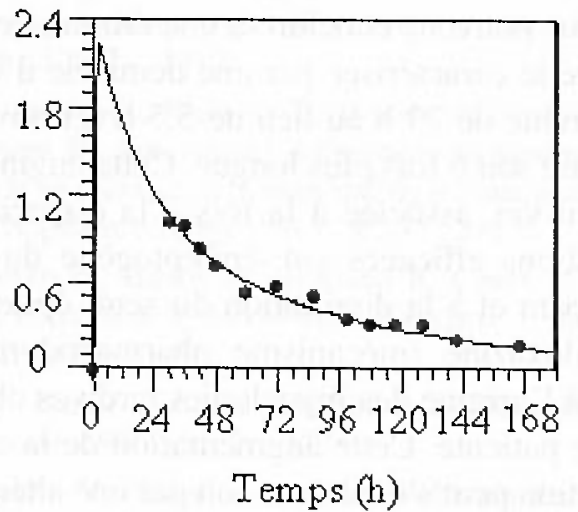

Figure 3 : a. Évolution des concentrations plasmatiques en fonction du temps de la viloxazine LP correspondant aux concentrations observées $(\bullet)$ et aux concentrations prédites (-).

b. Evolution des concentrations plasmatiques en fonction du temps du citalopram LP correspondant aux concentrations observées $(\bullet)$ et aux concentrations prédites (-).

Tableau II : Paramètres pharmacocinétiques de la viloxazine dans différentes études cliniques et toxicologiques en fonction de la dose et de la forme.

\begin{tabular}{|c|c|c|c|c|c|c|c|}
\hline $\mathbf{N}$ & Dose ingérée & $\underset{\text { (h) }}{\operatorname{Tmax}}$ & $\begin{array}{l}\mathrm{Cmax} \\
(\mathrm{mg} / \mathrm{l})\end{array}$ & $\begin{array}{l}\text { Demi-vie } \\
\text { (h) }\end{array}$ & $\begin{array}{c}\mathrm{Vd} / \mathrm{F} \\
\text { (I) }\end{array}$ & $\begin{array}{c}\mathrm{Cl} / \mathrm{F} \\
(\mathrm{ml} / \mathrm{min})\end{array}$ & Référence \\
\hline \multicolumn{8}{|c|}{ VILOXAZINE } \\
\hline 1 & $4,8 \mathrm{~g}$ & 36 & 60 & 29 & 1,05 & 26 & notre étude \\
\hline 12 & $300 \mathrm{mg} \mathrm{LP}$ & 4,5 & 1,49 & 7,1 & & & 25 \\
\hline 12 & $300 \mathrm{mg} \mathrm{LP}(28 \mathrm{j})$ & 5,3 & 2,9 & 5,6 & 1,1 & 164 & 26 \\
\hline 10 & $100 \mathrm{mg}$ & 1,7 & 2,2 & 3,5 & 0,8 & 167 & 10 \\
\hline 1 & $6 \mathrm{~g}$ & & 47 & & & & 20 \\
\hline \multicolumn{8}{|c|}{ CITALOPRAM } \\
\hline 1 & $? ?$ & 32 & 1,1 & 59 & & & notre étude \\
\hline
\end{tabular}


associant des troubles cardiaques, une agitation, des myoclonies tardives. L'absence de prélèvements concomitants de la prise médicamenteuse ne nous permet pas d'évaluer une éventuelle phase de distribution du citalopram. Seule sa demi-vie terminale a pu être déterminée. Chez notre patiente, la cinétique du citalopram est caractérisée par une élimination bi-exponentielle avec une demi-vie terminale de $59 \mathrm{~h}$, nettement supérieure à sa valeur théorique de $33 \mathrm{~h}$. La phase d'absorption est caractérisée par une concentration maximale de l'ordre de $2,2 \mathrm{mg} / \mathrm{l}$ et un temps maximal aux environs de $2 \mathrm{~h}$. L'administration de phénobarbital inducteur du métabolisme du citalopram (cytochrome P450 2C19), n'entraîne pas de diminution des concentrations plasmatiques de citalopram. En effet, le mécanisme d'induction enzymatique est un processus progressif dont l'effet maximal nécessite généralement un traitement de $7 \mathrm{j}$ par la molécule inductrice. Le caractère inducteur enzymatique du phénobarbital n'a pas eu le temps nécessaire à son expression. Pour la viloxazine LP, nous pouvons conclure à une élimination mono exponentielle caractérisée par une demi-vie d'élimination apparente de $29 \mathrm{~h}$ au lieu de 5,5 h retrouvée dans la littérature soit 6 fois plus longue. Cette augmentation de la demi-vie, associée à la fois à la disparition des concentrations efficaces anti-épileptogène du desmethyldiazépam et à la diminution du seuil épileptogène par la viloxażine (mécanisme pharmacodynamique) peut être à l'origine des myoclonies tardives observées chez cette patiente. Cette augmentation de la demi-vie d'élimination peut s'expliquer soit par une altération de la fonction rénale liée à l'arrêt cardio-respiratoire entrainnant une rhabdomyolyse et par la suite une insuffisance rénale aiguë, soit par une diminution du métabolisme hépatique. La concentration maximale estimée à $95 \mathrm{mg} / \mathrm{l}$ et la première concentration observée de $60 \mathrm{mg} / \mathrm{l}$ ont permis de confirmer a posteriori le diagnostic d'intoxication massive en viloxazine LP puis de calculer la dose supposée ingérée à plus de $4 \mathrm{~g}$. Cette concentration maximale atteinte probablement $12 \mathrm{~h}$ après l'ingestion pourrait être à l'origine de l'arrêt cardio-respiratoire. Ce taux sanguin mesurée de $60 \mathrm{mg} / \mathrm{L}$ est supérieur à̀ ceux décrits dans les cas de prise massive de la forme à libération immédiate (2426). Il est intéressant de noter la modification du profil pharmacocinétique de la viloxazine LP liée à une prise massive avec un allongement de la demi-vie d'élimination et une absorption ressemblant à celle de la forme à libération immédiate $(16,27,28)$. Selon la littérature, un lavage gastrique doit être réalisé le plus rapidement possible après une prise massive de viloxazine et une diurèse forcée permettrait de diminuer alors sa concentration plasmatique. Sur la courbe des concentrations de viloxazine LP en fonction du temps (Figure $2 a$ et 2b), nous pouvons constater la présence d'un phénomène de rebond de la concentration sur une période de 24 hi. Plusieurs hypothèses peuvent expliquer cet événement. Tout d'abord, un cycle entéro-hépatique et une absorption prolongée de la molécule, non décrit pour la forme LP dans la littérature $(16,29)$. Il a été montré chez le Rat et le Chien une excrétion biliaire avec une phase de réabsorption (30). Ce cycle n'a pas été étudié chez l'Homme et s'il existe, la réabsorption doit être importante puisque seulement $2 \%$ de la dose est éliminée dans les fécès. Cela pourrait être dû également à un phénomène de stase intestinale ou à une diminution de l'élimination rénale de la viloxazine ou à un phénomène de săturation métabolique de la viloxazine liée à la forte dose ingérée. Les signes cliniques cardiaques n'étaient pas présents lors de l'admission aux urgences, ce qui était dî probablement à une absorption retardée liée à la forme galénique et la forte dose ingérée. L'action conjointe de la viloxazine et du citalopram sur la fonction cardiaque conduisant à l'arrêt cardio-respiratoire dans les heures suivant la prise médicamenteuse permet de confirmer une modélisation satisfaisante de la cinétique des 2 produits. Cet arrêt cardio-respiratoire est renforcé par une dépression respiratoire due aux fortes concentrations de desméthyldiazépam. Bien que portant sur 13 points, il ne s'agit là que d'une estimation graphique des paramètres toxicocinétiques, ne permettant pas un parallélisme absolu avec les données de la littérature, notamment quant à la durée de la demivie d'élimination.

\section{Conclusion}

Ce cas de tentative de suicide par la viloxazine forme à libération prolongée associée au citalopram est une illustration des symptômes cliniques induits par une prise massive de cette classe d'antidépresseurs et du caractère cardiotoxique de la viloxazine. Cette toxicité tardive observée est en relation avec la prise massive de viloxazine forme LP. Les dosages effectués sur 13 prélèvements sanguins répartis sur $168 \mathrm{~h}$ nous ont permis d'effectuer un suivi toxicocinétique de la viloxazine et du citalopram. Ce suivi nous montre l'intérêt de la recherche en toxicologie d'urgence de ces nouveaux antidépresseurs non tricycliques devant un tableau clinique à composante cardiaque. En raison de leur prescription croissante, une recherche systématique de ces nouveaux antidépresseurs est donc nécessaire au vu des risques de cardiotoxicité. 


\section{Références}

1. Jackson W., Roose S., Glassman A. Cardiovascular toxicity and tricyclic antidepressants. Biomed Pharmacother. $1987 ; 41(7)$ : 377-82.

2. Harrigan R., Brady W. ECG abnormalities in tricyclic antidepressant ingestion. Am. J. Emerg. Med. 1999 ; 17(4) : 387-93.

3. Larkin G., Graeber G., Hollingsed M. Experimental amitriptyline poisoning: treatment of severe cardiovascular toxicity with cardiopulmonary bypass. Ann. Emerg. Med. $1994 ; 23(3): 480-6$.

4. Glauser J. Tricyclic antidepressant poisoning. Cleve. Clin. J. Med. 2000 ; 67(10) : 704-6, 709-13, 717-9.

5. Rudorfer M., Manji H., Potter W. Comparative tolerability profiles of the newer versus older antidepressants. Drug Saf. $1994 ; 10(1): 18-46$.

6. Dictionnaire Vidal en cours ou www.vidalpro.net

7. Micromedex Healthcare Serie.

8. Edwards J., Glen-Bott M. Does viloxazine have epileptogenic properties? J. Neurol. Neurosurg. Psychiatry. 1984; 47(9) : 960-4.

9. Meldrum B., Anlezark G., Adam H., Greenwood D. Anticonvulsant and proconvulsant properties of viloxazine hydrochloride: pharmacological and pharmacokinetic studies in rodents and the epileptic baboon Psychopharmacology. $1982 ; 76(3)$ : 212-7.

10. Vandel B., Vandel S., Allers G., Volmat R. Clinical pharmacology of viloxazine hydrochloride. Pharmacopsychiatria. 1981; 14(2) : 66-70.

11. Norman T., Burrows G., Davies B., Maguire K., Wurm J. Viloxazine plasma concentrations and clinical response. J. Affect. Disord. 1980 ; 2(3) : 157-64.

12. Investigator's brochure. Citalopram. H. Lundbeck, 4 june 1993

13. Bjerkenstedt L., Flyckt L., Overo K., Lingjaerde O. Relationship between clinical effects, serum drug concentration and serotonin uptake inhibition in depressed patients treated with citalopram. A double-blind comparison of three dose levels. Eur. J. Clin. Pharmacol. 1985 ; $28(5): 553-7$.

14. Kragh-Sorensen P., Overo K., Petersen O., Jensen K., Parnas W. The kinetics of citalopram: single and multiple dose studies in man. Acta Pharmacol. Toxicol. 1981 ; 48(1) : 53-60.

15. Vandel B., Vandel S., Jounet J., Blum D. Pharmacokinetics of viloxazine hydrochloride in man. Eur. J. Drug Metab. Pharmacokinet. $1982 ; 7(1): 65-8$.
16. Kergueris M., Bourin M., Ribeyrol M., Beneroso N., Normand Y., Larousse C. Comparative pharmacokinetic study of conventional and sustained-release viloxazine in normal volunteers. Neuropsychobiology. $1989 ; 20(3)$ : $136-40$.

17 Dossier technique du Seropram ${ }^{\circledR}$.

18 Uges D.R.A.. Therapeutic and toxic drug concentrations. Tiaft Bulletin. 1996 ; 26(1- suppl.) : 1-34.

19 Fiche technique SFTA TOX 190, antidépresseurs non-imipraminiques non-IMAO (toxicologie clinique), www.sfta.org

20 Deveaux M., Ferroul D., Leman C., Tournel G., Hédouin V., Gosset D. Forensic intoxications by new antidepressants: report of 22 cases. Ann. Toxicol. Anal. $2002 ; 15(3)$ : 78

21. Falcy M., Riboulet-Delmas G., Efthymiou M. Acute poisoning by viloxazine chlorhydrate taken by itself. Encéphale.1983; 9(2) : 137-44

22. Faller J., Feissel M., Ruyer O., Louis J., Picard A., Simon G. Cardiogenic shock status with conductive disorders in severe poisoning with viloxazine: A case. Presse Med. $1988 ; 17(27): 1412$.

23. Altamura AC., Melorio T., Invernizzi G., Colacurcio F., Gomeni R. Age-related differences in kinetics and sideeffects of viloxazine in man and their clinical implications. Psychopharmacology. 1983 ; 81(4) : 281-5.

24. Brosnan R., Busby A., Holland R. Cases of overdosage with viloxazine hydrochloride (Vivalan). J. Int. Med. Res. $1976 ; 4(2): 83-5$.

25. Py P., Danel V. Acute agitation and viloxazine overdose. Therapie. $1996 ; 51(3): 327$

26. Regouby Y. Posterior coronary ischemia during massive viloxazine and clotiazepam poisoning. Therapie. 1991 ; 46(5) : 409-10.

27. Norris S., Harry J., Adam H. "Vivalan". A bioavailabilty study comparing two slow-release formulations with standard formulation in healthy volunteers. Confidential Report, June 1986. ICI-Pharma laboratories.

28. Bouquet S., Guibert S., Lavoisy J. Oral pharmacokinetics of a new slow-release formulation of viloxazine in depressed patients. Psychopharmacology. $1989 ; 96: 144$

29. Case D., Reeves P. The disposition and metabolism of I.C.I. 58,834 (viloxazine) in humans. Xenobiotica. 1975 ; $5(2): 113-29$.

30. Case D., Illston H., Reeves P., Shuker B., Simons P. The disposition and metabolism of I.C.I. 58,834 (viloxazine) in animals. Xenobiotica. 1975 ; 5(2) : 83-111. 